\title{
Geometrical Methods in Goursat Categories
}

\author{
Brice Réné Amougou Mbarga
}

University of Yaoundé I, Laboratory of Algebra, Geometry and Application,

P.O.Box: 812, Yaoundé, Cameroon; e-mail: renebrice3@gmail.com

\begin{abstract}
The main aim of the paper is to show that the Little Desarguesian Theorem, the Escher Cube, Closure Lemma 1 and 3, hold in any regular Mal'tsev categories. We prove that Mal'tsev categories may be characterized through variations of the Little Desarguesian Theorem, the Escher Cube, Closure Lemma 1 and 3, that is classically expressed in terms of four congruences $R, S_{1}, S_{2}$ and $T$, and characterizes congruence modular varieties. The proof of this result in a varietal context may be obtained exclusively through the Little Desarguesian Theorem, the Escher Cube, Closure Lemma 1 and 3. This was shown by H.P. Gumm in Geometric Methods in Congruence Modular Algebras. We prove that for any $2 n+1$-permutable category $\mathcal{E}$, the category $\operatorname{Equiv}(\mathcal{E})$ of equivalence relations in $\mathcal{E}$ is also a $2 n+1$-permutable category.
\end{abstract}

\section{Introduction}

The study of Mal'tsev categories originates from a classical theorem of Mal'tsev in 1954 [5]. For a variety of universal algebras $\mathcal{V}$ (i.e., a category of models of a finitary one-sorted algebraic theory), he proved that the following conditions are equivalent:

(i) for any pair of congruences $R$ and $S$ on the same algebra $X$ in $\mathcal{V}$, the equality $R S=S R$ holds;

(ii) the algebraic theory of $\mathcal{V}$ contains a ternary operation $p$ satisfying the equations

$$
\left\{\begin{array}{l}
p(x, y, y)=x, \\
p(x, x, y)=y .
\end{array}\right.
$$

Received: February 28, 2021; Accepted: April 24, 2021

2010 Mathematics Subject Classification: 05C05.

Keywords and phrases: Mal'tsev, Goursat, Shifting Lemma, Little Desarguesian, Escher Cube, Little Pappian Theorem. 
Varieties satisfying these conditions are now commonly called Mal'tsev varieties [5] (or 2-permutable varieties), and such a term $p$ a Mal'tsev operation.

One of the results that A. Carboni, J. Lambek, M. C. Pedicchio had in mind in [5] was the extension to Mal'tsev categories of the Goursat Lemma, due to E. Goursat and stated in the category of groups as follows:

Lemma 1.1. (Goursat Lemma) Let $L$ and $R$ be arbitrary groups. Then there is a bijection between the set $\mathcal{S}$ of all subgroups of $L \times R$ and the set $\mathcal{T}$ of all quintuple $(A, B, C, D, \theta)$, where $B \unlhd A \leq L, D \unlhd C \leq R$ and $\theta: A / B \rightarrow C / D$ is a bijective homomorphism (here $\leq$ denotes subgroup and $\unlhd$ denotes normal subgroup). More precisely, the subgroup corresponding to $(A, B, C, D, \theta)$ is

$$
G=\{(g, h) \in A \times C: \theta(g B)=h D\} .
$$

This result was used to obtain general forms of the Zassenhaus lemma and the Jordan-Hölder-Schreier theorem for normal series in [12] and was generalized to Mal'tsev varieties by J. Lambek in [12]. After an in-depth analysis, A. Carboni, J. Lambek and M. C. Pedicchio proved that this result of E. Goursat holds not only in the Mal'tsev categories but also in those regular categories wherein each relation $P$ from $A$ to $B$ satisfies the equality

$$
P P^{o} P P^{o}=P P^{o}
$$

That is how Goursat categories were born: these are the regular categories in which each relation $P$ satisfies the condition (1.1). In 1993, A. Carboni, M. Kelly and M. C. Pedicchio observed in [8] that the condition $[1.1$ is in fact strictly weaker than the difunctionality $P P^{o} P=P$ equivalent to the Mal'tsev condition $R S=S R$ in regular categories. They also observed that condition (1.1) is equivalent in a regular category to the 3-permutability of equivalence relations

$$
R S R=S R S,
$$

for any pair of equivalence relations $R$ and $S$ on the same object. 


\section{Structure of the paper}

In Section 2 we introduce the basic categorical notions and results we shall need in the following section. In particular we recall the relationship between some epimorphisms such as strong epimorphisms, regular epimorphisms and split epimorphisms. We then recall some basics facts about regular categories and relations in regular categories. We also recall the notion Barr's Metatheorem concerning the internal logic of regular categories.

In Sections 3 and 4 the main results of the present paper show that stronger versions of the Little Desarguesian Theorem, the Escher Cube, Closure Lemma 1 and 3, characterize regular categories that are Mal'tsev categories and that are Goursat categories.

In Section 5 we prove that for any $2 n+1$-permutable category $\mathcal{E}$, the category $\operatorname{Equiv}(\mathcal{E})$ of equivalence relations in $\mathcal{E}$ is also a $2 n+1$-permutable category.

\section{Preliminaries}

In this section, we recall some elementary categorical notions and properties needed in the subsequent sections.

\subsection{Regular epimorphisms}

In this subsection, we examine various types of epimorphisms in order to understand the notions of regular categories.

Definition 2.1. A morphism $f: A \rightarrow B$ in a category $\mathcal{E}$ is an epimorphism if, for any pair of parallel arrows $u, v: B \rightarrow C$ such that $u \circ f=v \circ f$, one has $u=v$.

In the category Set of sets, the epimorphisms are precisely the surjective maps. In the category Grp and $\mathbf{A b}$ of abelian groups, the epimorphisms are the surjective homomorphisms. The notion of monomorphism is defined dually:

Definition 2.2. A morphism $f: A \longmapsto B$ in a category $\mathcal{E}$ is a monomorphism if, for any pair of parallel arrows $u, v: C \rightarrow A$ such that $f \circ u=f \circ v$, one has $u=v$. 
Monomorphisms in Top (the category of topological spaces) are the continuous injective maps.

Definition 2.3. An arrow $f: A \rightarrow B$ in a category $\mathcal{E}$ is a strong epimorphism if, given any commutative square

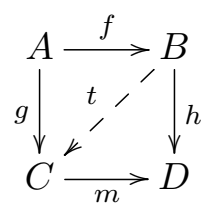

where $m: C \longmapsto D$ is a monomorphism, there exists a unique arrow $t: B \rightarrow C$ such that $m \circ t=h$ and $t \circ f=g$.

Strong epimorphisms have the following properties:

(i) if $\alpha: A \rightarrow B$ and $\beta: B \rightarrow C$ are two strong epimorphisms, then $\beta \alpha: A \rightarrow C$ is a strong epimorphism.

(ii) if $\alpha: A \rightarrow B$ and $\beta: B \rightarrow C$ are such that $\beta \alpha: A \rightarrow C$ is a strong epimorphism, then $\beta: B \rightarrow C$ is a strong epimorphism.

Definition 2.4. An arrow $f: A \rightarrow B$ is a regular epimorphism if it is the coequalizer of two arrows in $\mathcal{E}$. In other words, $f$ is a regular epimorphism if one can find $u ; v \in \mathcal{E}$ such that $f=\operatorname{Coeg}(u, v)$.

Definition 2.5. A split epimorphism is an arrow $f: A \rightarrow B$ such that there is an arrow $i: B \rightarrow A$ with $f \circ i=1_{B}$.

In any category $\mathcal{E}$, the following properties hold.

(i) every split epimorphism is regular;

(ii) every regular epimorphism is strong. 


\subsection{Regular categories and relations}

Recall that a kernel pair of a morphism $f: A \rightarrow B$ is a pair of parallel morphisms $E q(f) \stackrel{f_{1}}{\longrightarrow} A$ obtained via the following pullback

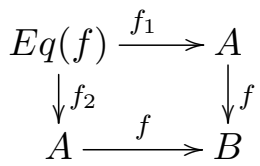

Let $f: A \rightarrow B$ be a map in Set, and $E q(f)=\left\{(x, y) \in A^{2} / f(x)=f(y)\right\}$ its kernel pair.

Definition 2.6. [13] A finitely complete category $\mathcal{E}$ is regular when

(a) $\mathcal{E}$ has coequalisers of kernel pairs;

(b) regular epimorphisms are stable under pullbacks in $\mathcal{E}$ : That is, given a pullback diagram

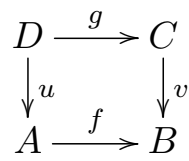

where $f$ is regular epimorphism, then $g$ is a regular epimorphism.

Example 2.7. Regular categories, even complete ones are abundant. The category of models of any equational theory is complete and regular. This includes the categories of sets, lattices, groups, rings, etc. The category of compact Hausdorff spaces is complete regular as is every abelian category. Also the category opposite to the category of sets is regular.

This definition of regular category is equivalent with the following one: any morphism $f$ admits a (unique up to isomorphism) factorization $f=m r$, where $r$ is a regular epimorphism and $m(=\operatorname{Imf})$ is a monomorphism, and these factorizations are pullback stable.

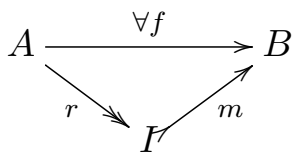


If $R$ is a relation from $A$ to $B$, namely a subobject $\left(r_{1}, r_{2}\right): R \longmapsto A \times B$, its opposite relation, denoted $R^{\circ}$, is a relation from $B$ to $A$, the subobject $\left(r_{2}, r_{1}\right): R \longmapsto B \times A$. Given another relation $\left(s_{1}, s_{2}\right): S \longmapsto B \times C$ from $B$ to $C$, On first builds the pullback

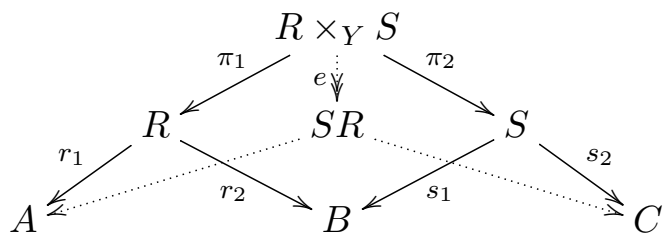

We obtain a composite relation $\operatorname{Im}\left(r_{1} \pi_{1}, s_{2} \pi_{2}\right): S R \rightarrow A \times C$.

Definition 2.8. [26] Let $\mathcal{E}$ be a category with finite limits.

(i) A relation $R \longmapsto A \times A$ is reflexive when there is an arrow $\delta: X \rightarrow R$ such that $d_{1} \circ \delta=1_{X}=d_{2} \circ \delta$, or $1_{A} \leq R$

(ii) $R$ is symmetric if there is an arrow $\sigma: R \rightarrow R$ such that $d_{1} \circ \sigma=d_{2}$ and $d_{2} \circ \sigma=d_{1}$, or $R^{\circ} \leq R$

(iii) Consider the pullback

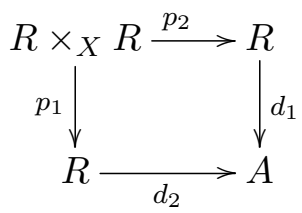

the relation $R$ is transitive if there is an arrow $\tau: R \times_{X} R \rightarrow R$ such that $d_{1} \circ \tau=$ $d_{1} \circ p_{1}$ and $d_{2} \circ \tau=d_{2} \circ p_{2}$ or $R R \leq R$.

An (internal) equivalence relation is a reflexive, symmetric and transitive relation.

In particular, the kernel pair $\left\langle f_{1}, f_{2}\right\rangle: E q(f) \rightrightarrows A \times A$ of a morphism $f: A \rightarrow B$ (obtained by building the pullback of $f$ along $f$ ) is an equivalence relation. The equivalence relations that occur as kernel pairs of some morphism in a category $\mathcal{E}$ are called effective.

We denoted by $\operatorname{Equiv}(\mathcal{E})$ the category whose objects are equivalence relations in $\mathcal{E}$ and arrows from $\left\langle r_{1}, r_{2}\right\rangle: R \longmapsto A \times A$ to $\left\langle s_{1}, s_{2}\right\rangle: S \longmapsto B \times B$ are pairs $(f, g)$ of arrows in $\mathcal{E}$ making the following diagram commute 


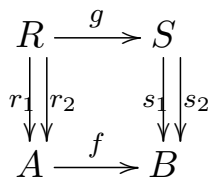

$s_{1} g=f r_{1}$ and $s_{2} g=f r_{2}$.

Definition 2.9. Let $\mathcal{E}$ be a regular category, $\left(R, r_{1}, r_{2}\right)$ a relation on $A$ and $f: A \rightarrow B$ a regular epimorphism. We define the regular image of $R$ along $f: A \rightarrow B$ to be the relation $f(R)$ on $B$ induced by the (regular epimorphism, monomorphism) factorization $\left\langle s_{1}, s_{2}\right\rangle \psi$ of the composite $(f \times f)\left\langle r_{1}, r_{2}\right\rangle$ :

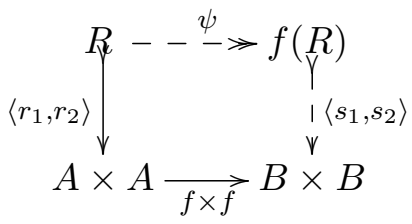

Note that the regular image $f(R)$ can be obtained as the relational composite $f(R)=f R f^{o}=f r_{2} r_{1}^{o} f^{o}$. When $R$ is an equivalence relation, $f(R)$ is also reflexive and symmetric.

\subsection{Barr's embedding Metatheorems}

The following celebrated lemma is crucial for the whole of category theory.

Lemma 2.10. (Yoneda lemma) Let $G: \mathcal{E} \rightarrow$ Set be a functor defined on an arbitrary category $\mathcal{E}$. For every object $C \in \mathcal{E}$, there exists a bijection $F(C) \cong \operatorname{Nat}(\mathcal{E}(C,-), F)$ between the set $F(C)$ and the set of natural transformations from the representable functor $\mathcal{E}(C,-)$ to the functor $F$. These bijections are natural both in $C$ and in $F$. Then given two objects $C, D \in \mathcal{E}$, there are bijections

$$
\mathcal{E}(D, C) \cong \operatorname{Nat}(\mathcal{E}(C,-), \mathcal{E}(D,-))
$$

and

$$
\mathcal{E}(C, D) \cong \operatorname{Nat}(\mathcal{E}(-, C), \mathcal{E}(-, D)) .
$$


All this allows defining the "Yoneda embedding of $\mathcal{E}$ " [6] which is the functor

$$
Y_{\mathcal{E}}: \mathcal{E} \longrightarrow\left[\mathcal{E}^{o p}, \text { Set }\right], C \longmapsto \mathcal{E}(-, C) \text {. }
$$

This functor $Y_{\mathcal{E}}$ is a full and faithful embedding of $\mathcal{E}$ in the category $\left[\mathcal{E}^{o p}\right.$, Set $]$ of contravariant functors from $\mathcal{E}$ to Set and natural transformations between them.

These well-known facts allow at once a powerful set-theoretical-like technique for proving various results in a category by just proving them in the category of sets.

Metatheorem 2.11. For every small regular category $\mathcal{E}$ there is a small category $\mathcal{D}$ and a full and faithful embedding

$$
Z: \mathcal{E} \rightarrow\left[\mathcal{D}^{o p}, \text { Set }\right]
$$

which preserves and reflects finite limits and regular epimorphisms.

Metatheorem 2.12. Let $P$ be a statement of the form $a \Longrightarrow b$, where $a$ and $b$ can be expressed as conjunctions of properties in the following list:

(i) some finite diagram is commutative;

(ii) some morphism is a monomorphism;

(iii) some morphism is an isomorphism;

(iv) some finite diagram is a limit diagram;

( $v$ ) some arrow $f: A \longrightarrow B$ factors (of course, uniquely) through some specified monomorphism $s: S \longrightarrow B$.

If this statement $P$ is valid in the category of sets, it is valid in every category.

\subsection{Shifting Lemma, Little Desarguesian Theorem, Little Pappian Theorem, Escher Cube, Closure Lemma 1 and 3}

For a variety $\mathcal{V}$ of universal algebras, Gumm's Shifting Lemma [16] is stated as follows. Given congruences $R, S_{1}$ and $T$ on the same algebra $X$ in $\mathcal{V}$ such that $R \wedge S_{1} \leqslant T$, whenever $x, y, t, z$ are element in $X$ with $(x, y) \in R \wedge T,(x, t) \in S_{1},(y, z) \in S_{1}$ and 
$(t, z) \in R$, it then follows that $(t, z) \in T$ :

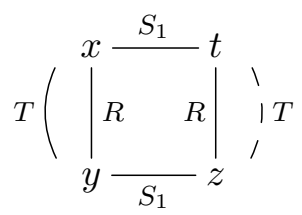

Closure Lemma 1:

A variety $\mathcal{V}$ of universal algebras satisfies the Closure Lemma 1, [27] if given congruences $R, S_{i}$ and $T$ on the same algebra $X$ in $\mathcal{V}$ such that $R \wedge S_{i} \leqslant T$, whenever $x, y, z, x^{\prime}, y^{\prime}, z^{\prime}, u, u^{\prime}$ are element in $X$ with $(x, z),\left(x^{\prime}, z^{\prime}\right),(u, y),\left(u^{\prime}, y^{\prime}\right) \in$ $R, \quad\left(z, z^{\prime}\right),\left(x^{\prime}, x\right),\left(u, u^{\prime}\right),\left(y, y^{\prime}\right) \in S_{1},(z, u),(x, y) \in S_{2}$ and $\left(z^{\prime}, u^{\prime}\right) \in T$, it then follows that $\left(x^{\prime}, y^{\prime}\right) \in T$ we have

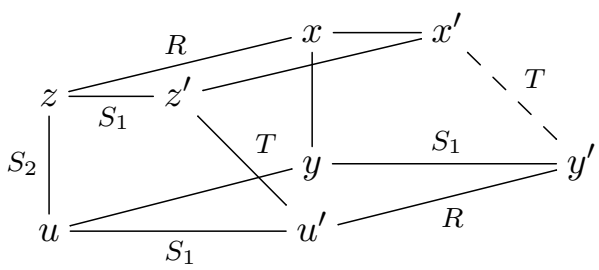

Mainly we are interested in the following two special case. Firstly, letting $x=x^{\prime}$ and $z=z^{\prime}$ we obtain:

A variety $\mathcal{V}$ of universal algebras satisfies the Little Desarguesian Theorem [27] if given congruences $R, S_{i}$ and $T$ on the same algebra $X$ in $\mathcal{V}$ such that $R \wedge S_{i} \leqslant T$, whenever $x, y, z, x^{\prime}, y^{\prime}, z^{\prime}$ are element in $X$ with $(x, z),\left(x^{\prime}, z^{\prime}\right) \in S_{1},(z, y),\left(z^{\prime}, y^{\prime}\right) \in$ $S_{2},\left(z, z^{\prime}\right),\left(x, x^{\prime}\right),\left(y, y^{\prime}\right) \in R$ and $(x, y) \in T$, it then follows that $\left(x^{\prime}, y^{\prime}\right) \in T$ :

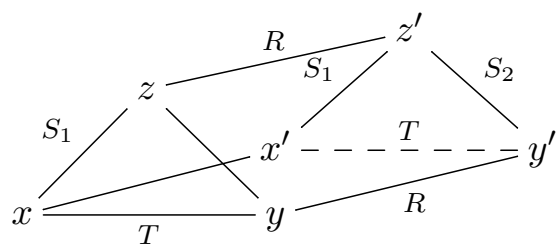

Escher Cube or Closure Lemma 2 :

A variety $\mathcal{V}$ of universal algebras satisfies the Closure Lemma 2, [27] if given congruences $R, S_{i}$ and $T$ on the same algebra $X$ in $\mathcal{V}$ such that $R \wedge S_{i} \leqslant T$, 
whenever $x, y, z, x^{\prime}, y^{\prime}, z^{\prime}, u, u^{\prime}$ are element in $X$ with $\left(y, y^{\prime}\right),\left(u^{\prime}, u\right),\left(z, z^{\prime}\right),\left(x, x^{\prime}\right) \in$ $S_{1},(u, y),(x, z) \in S_{2},\left(y^{\prime}, u^{\prime}\right),\left(x^{\prime}, z^{\prime}\right) \in R$ and $\left(u^{\prime}, z^{\prime}\right) \in T$, one has $\left(x^{\prime}, y^{\prime}\right) \in T$ :

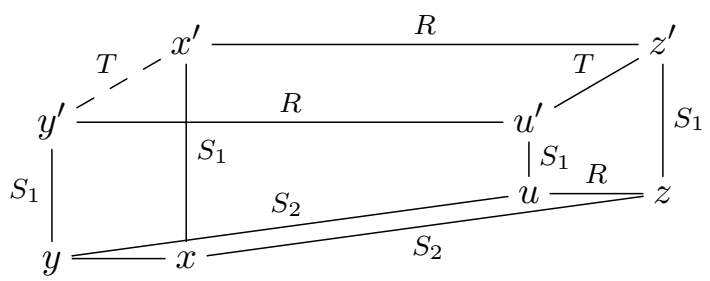

The little Pappian Theorem

A variety $\mathcal{V}$ of universal algebras satisfies the little Pappian Theorem, [27] if given congruences $R, S_{i}$ and $T$ on the same algebra $X$ in $\mathcal{V}$ such that $R \wedge S_{i} \leqslant T$, whenever $x, y, u, z, x^{\prime}, y^{\prime}, z^{\prime}$ are element in $X$ with $\left(u, y^{\prime}\right),(x, z) \in S_{1},\left(x^{\prime}, x\right),\left(u, z^{\prime}\right) \in$ $S_{2},\left(x^{\prime}, u\right),(u, z),\left(y^{\prime}, x\right),\left(x, z^{\prime}\right) \in R$ and $\left(z, z^{\prime}\right) \in T$, then $\left(x^{\prime}, y^{\prime}\right) \in T$ :

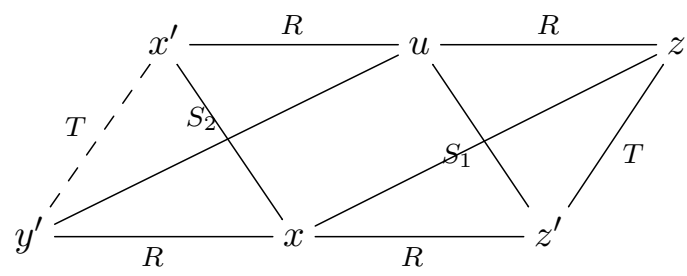

Similarly, on identifying $S_{2}$ with $T$ and $u$ with $z^{\prime}$ we obtain:

A variety $\mathcal{V}$ of universal algebras satisfies the Closure Lemma 3 if given congruences $R, S$ and $T$ on the same algebra $X$ in $\mathcal{V}$ such that $R \wedge S \leqslant T$, one has

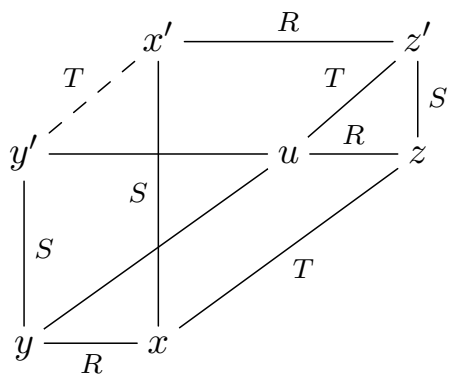




\section{Regular Mal'tsev Categories}

A finitely complete category $\mathcal{E}$ is called a Mal'tsev category if every reflexive relation in $\mathcal{E}$ is an equivalence relation.

Example 3.1. (1) Groups, Rings and Modules are Mal'tcev categories;

(2) abelian categories are Mal'tcev categories;

(4) any slice of a Malcev category.

These categories are also characterized by other properties on relations, as follows:

Theorem 3.2. $[15]$ Let $\mathcal{E}$ be a regular category. The following statements are equivalent:

(i) $\mathcal{E}$ is a Mal'tsev category;

(ii) $\forall R, S \in \operatorname{Equiv}(X), R \vee S=R S=S R \in \operatorname{Equiv}(X)$, for any $X$;

(iii) every reflexive relation $E$ in $\mathcal{E}$ is symmetric: $E^{\circ}=E$;

(iv) any relation $D$ is difunctional: $D D^{o} D=D$;

$(v)$ every reflexive relation $T$ in $\mathcal{E}$ is transitive: $T T=T$.

Theorem 3.3. Let $\mathcal{E}$ be a regular Mal'tsev category. Then

(1) the Little Desarguesian Theorem holds true in $\mathcal{E}$.

(2) the Escher Cube holds true in $\mathcal{E}$;

(3) the Closure Lemma 1 holds true in $\mathcal{E}$;

(4) the Closure Lemma 3 holds true in $\mathcal{E}$;

(5) the Little Pappian Theorem holds true in $\mathcal{E}$.

Proof. In a regular context, it suffices to give a proof in set-theoretical terms (see Metatheorem 2.12 and [27], for instance). We show that the Little Desarguesian Theorem holds true in $\mathcal{E}$.

Let $R, S_{1}, S_{2}$ and $T$ be equivalence relations on the same object $X$ in $\mathcal{E}$ such that $R \wedge S_{i} \leqslant T$ and assume that $x, y, z, x^{\prime}, y^{\prime}, z^{\prime}$, are related as in 2.2. The pairs 
$(y, y),\left(y^{\prime}, y^{\prime}\right),(x, z),\left(x^{\prime}, z^{\prime}\right)$ are elements of $S_{1}$. Defining equivalence relations $P, Q$ and $F$ on $S_{1}$ by:

$$
\begin{aligned}
(u, v) P(r, s) & \Leftrightarrow v S_{2} s \\
(u, v) Q(r, s) & \Leftrightarrow u T r \text { and } v S_{2} s \\
(u, v) F(r, s) & \Leftrightarrow u R r \text { and } v R s
\end{aligned}
$$

We obtain

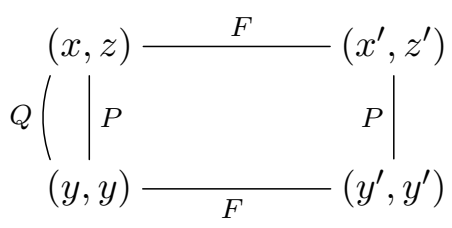

If we want to apply the Shifting Lemma we have to check that $F \wedge P \leq Q$. Thus, let $(a, b) F \wedge P(c, d)$. First of all $(a, b) \in S_{1}$ and $(c, d) \in S_{1}$. Moreover $(b, d) \in R \wedge S_{2}$. Hence $(b, d) \in T$ by assumption. Hence

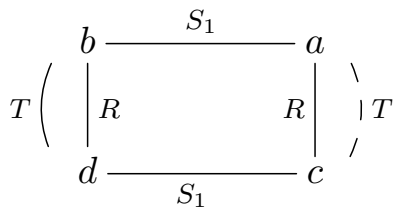

We may apply the Shifting Lemma to

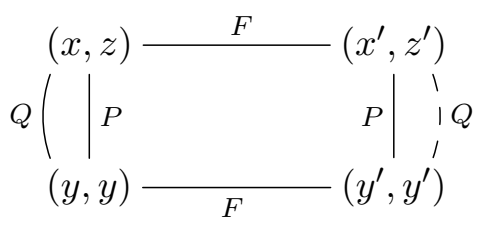

We conclude that $\left(x^{\prime}, z^{\prime}\right) Q\left(y^{\prime}, y^{\prime}\right)$ and, consequently, that $\left(x^{\prime}, y^{\prime}\right) \in T$.

Similarly we show that the Closure Lemma 1 holds true in $\mathcal{E}$;

Let $R, S_{1}, S_{2}$ and $T$ be equivalence relations on the same object $X$ in $\mathcal{E}$ such that $R \wedge S_{i} \leqslant T$ and assume that $x, y, z, x^{\prime}, y^{\prime}, z^{\prime}$, are related as in 2.1. The pairs 
$\left(x, x^{\prime}\right),\left(y, y^{\prime}\right),\left(z^{\prime}, z\right),\left(u, u^{\prime}\right)$ are elements of $S_{1}$. We obtain

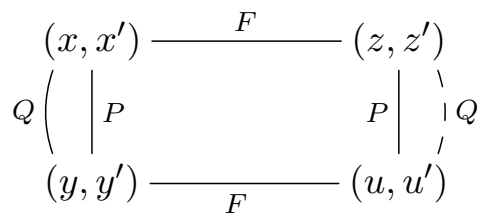

Similarly we can show $(3), \cdots,(5)$.

Using the fact that in a Mal'tsev category reflexive relations coincide with equivalence relations, or with symmetric relations, we are now going to show that regular Mal'tsev categories may be characterized through a stronger version of the diagram 2.2] where, in the assumption, the equivalence relations are replaced by reflexive relations. Note that, for diagram such as (2.2) where $R, S$ or $T$ are not symmetric, the relations are always to be considered from left to right and from top to bottom. To avoid ambiguity with the interpretation of such diagrams, from now on we will write $a \stackrel{E}{\longrightarrow} b$ to mean that $(a, b) \in E$ whenever $E$ is a non-symmetric relation.

Theorem 3.4. Let $\mathcal{E}$ be a regular category. The following conditions are equivalent:

(1) $\mathcal{E}$ is a Mal'tsev category;

(2) the Little Desarguesian Theorem holds in $\mathcal{E}$ when $R, T, S_{1}, S_{2}$ are reflexive relations on $X$;

(3) the Escher Cube holds in $\mathcal{E}$ when $R, T, S_{1}, S_{2}$ are reflexive relations on $X$;

(4) the Closure Lemma 1 holds in $\mathcal{E}$ when $R, T, S_{1}, S_{2}$ are reflexive relations on $X$.

(5) the Little Pappian Theorem holds true in $\mathcal{E}$ when $R, T, S_{1}, S_{2}$ are reflexive relations on $X$;

(6) the Closure Lemma 3 holds true in $\mathcal{E}$ when $R, T, S_{1}, S_{2}$ are reflexive relations on $X$.

Proof. (1) $\Rightarrow(2)$ : Since $\mathcal{E}$ is a Mal'tsev category, reflexive relations are necessarily equivalence relations. Since by Theorem 3.2, the diagram (2.2) holds for any reflexive 
relations in $\mathcal{E}$.

$(2) \Rightarrow(1):$ We shall prove that every reflexive relation $\left\langle e_{1}, e_{2}\right\rangle: E \mapsto X \times X$ is symmetric (Theorem 3.2(iii)). Suppose that $(x, y) \in E$, and consider the reflexive relations $T$ and $R$ on $E$ defined by the following pullbacks:
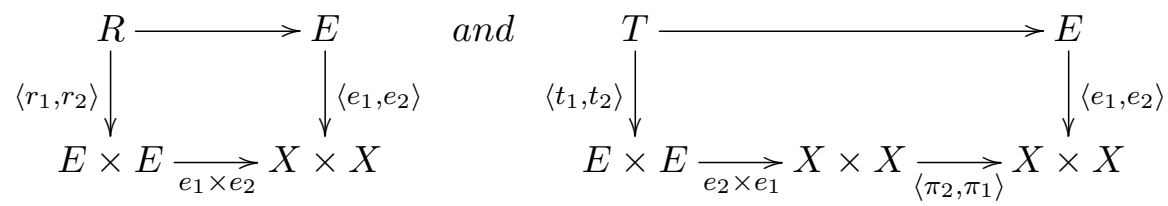

We have

$$
(a E b, c E d) \in R \text { if and only if }(a, d) \in E \text {, }
$$

and

$$
(a E b, c E d) \in T \text { if and only if }(c, b) \in E \text {. }
$$

The reflexive relation on $E$ we consider are the kernel pair $E q\left(e_{1}\right), E q\left(e_{2}\right)$ of $e_{1}$ and $e_{2}$ respectively.

$E q\left(e_{1}\right), E q\left(e_{2}\right)$, are equivalence relation, with the property that $E q\left(e_{i}\right) \leqslant R$ and $E q\left(e_{i}\right) \leqslant T$, so that $R \wedge E q\left(e_{i}\right)=E q\left(e_{i}\right) \leqslant T, i=1,2$. We may apply the assumption to the following relations given in solid lines

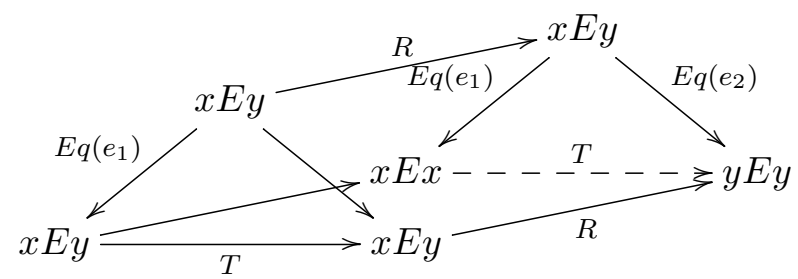

( $x E x$ and $y E y$ by the reflexivity of the relation $E)$. We conclude that $(x E x, y E y) \in T$ and, consequently, that $(y, x) \in E$.

The proof of $(1) \Leftrightarrow(3)$ is similar. Since the Little Desarguesian Theorem and the Closure Lemma 2 are immediate consequences of Closure Lemma 1 with $x=x^{\prime}$ and $z=z^{\prime}$. 
$(3) \Rightarrow(1)$ : evident, $(3) \Rightarrow(1)$ With the assumption of previous proof, we obtain

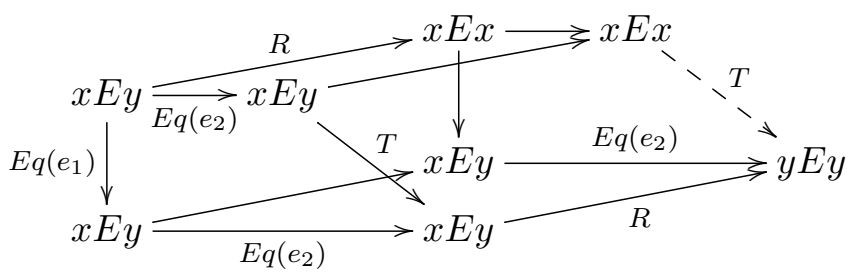

We conclude that $(x E x, y E y) \in T$, then $(y, x) \in E$. (5) $\Rightarrow(1)$ obvious, $(5) \Rightarrow(1)$ we observe with $S_{1}=E q\left(e_{2}\right)$ and $S_{2}=E q\left(e_{1}\right)$

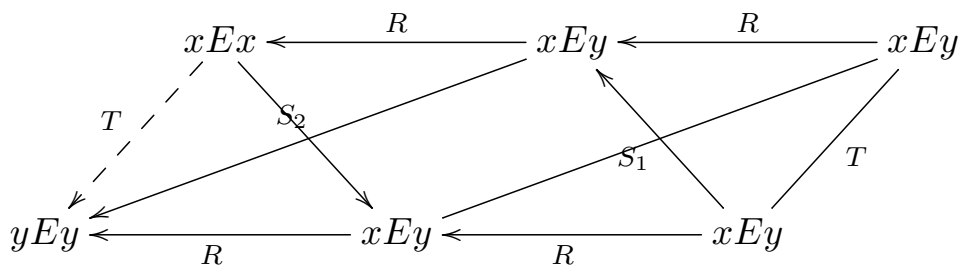

$(6) \Rightarrow(1)$ : we observe with $S=E q\left(e_{2}\right)$

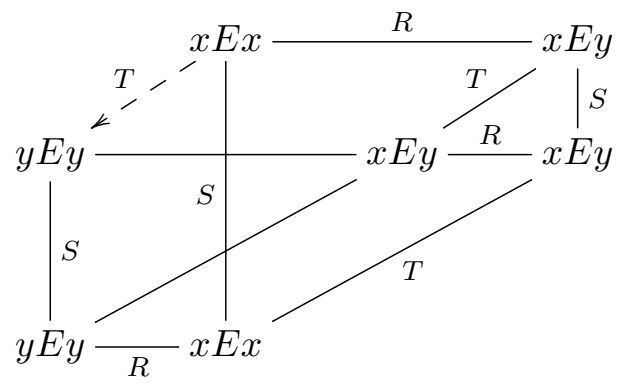

Corollary 3.5. Let $\mathcal{E}$ be a regular category. The following conditions are equivalent:

(1) $\mathcal{E}$ is a Mal'tsev category;

(2) the Little Desarguesian Theorem holds in $\mathcal{E}$ when $R, T$, are reflexive relations and $S_{1}, S_{2}$ are equivalence relations on $X$; 
(3) the Escher Cube holds in $\mathcal{E}$ when $R, T$, are reflexive relations and $S_{1}, S_{2}$ are equivalence relations on $X$;

(4) the Closure Lemma 1 holds in $\mathcal{E}$ when $R, T$, are reflexive relations and $S_{1}, S_{2}$ are equivalence relations on $X$;

(5) the Little Pappian Theorem holds true in $\mathcal{E}$ when $R, T$, are reflexive relations and $S_{1}, S_{2}$ are equivalence relations on $X$;

(6) the Closure Lemma 3 holds true in $\mathcal{E}$ when $R, T$, are reflexive relations and $S_{1}, S_{2}$ are equivalence relations on $X$.

A variety of algebras $\mathcal{V}$ is a majority category if and only if it admits a majority term, i.e., a ternary term $m(x, y, z)$ satisfying the equations:

$$
\begin{aligned}
& m(x, x, y)=x, \\
& m(x, y, x)=x, \\
& m(y, x, x)=x .
\end{aligned}
$$

For a proof of this statement, we refer the reader to [23].

Example 3.6. $(i)$ A subvariety $\mathcal{V}$ of the variety of rings is a majority category if and only if $\mathcal{V}$ satisfies the equation $x^{n}=x$ for some $n>2$. In particular, the category BoRg of Boolean rings is a majority category.Then the polynomial

$$
m(x, y, z)=x-(x-y)(x-z)^{n-1},
$$

is a majority term for $\mathcal{V}$.

(ii) The category NReg of von Neumann regular rings (see [21] ) is the class of all rings $R$ such that for any $a \in R$ there exists $x \in R$ such that $a=a x a$. The category NReg is a majority category.

Suppose that $A, B, C$ are rings and that $R$ is a subring of $A \times B \times C$ which is a von Neumann regular ring. Let $\bar{a}=\left(a, b, c^{\prime}\right), \bar{b}=\left(a, b^{\prime}, c\right), \bar{c}=\left(a^{\prime}, b, c\right)$ be any elements of $R$. Then since $R$ is von Neumann regular, there exists $x=\left(x_{1}, x_{2}, x_{3}\right) \in R$ such that

$$
(\bar{a}-\bar{b}) x(\bar{a}-\bar{b})=(\bar{a}-\bar{b}) .
$$


Then it is easy to see that

$$
\bar{a}-(\bar{a}-\bar{b}) x(\bar{a}-\bar{c})=(a, b, c)
$$

so that $(a, b, c) \in R$.

For a regular category $\mathcal{E}$, the property of being a majority category can be equivalently defined as follows (see [21]): for any reflexive relations $R, S$ and $T$ on the same object $X$ in $\mathcal{E}$, the inequality

$$
R \wedge(S T) \leqslant(R \wedge S)(R \wedge T)
$$

holds.

Lemma 3.7. Let $\mathcal{E}$ be a majority category $\mathcal{E}$. Then

(i) The Little Desarguesian Theorem holds true in $\mathcal{E}$;

(ii) Escher Cube holds true in $\mathcal{E}$;

(iii) Closure Lemma 1 holds true in $\mathcal{E}$;

(iv) Closure Lemma 3 holds true in $\mathcal{E}$.

Proof. The proof of this result is based on the fact that a majority category satisfies the Shifting Lemma.

\section{Regular Goursat Categories}

A regular category $\mathcal{E}$ is called a Goursat category [14] when it is 3-permutable, i.e. for any pair of equivalence relations $R$ and $S$ on the same object in $\mathcal{E}$ one has $R S R=S R S$.

Example 4.1. Mal'tsev categories.

As examples of Goursat categories that are not regular Mal'tsev categories we have the category of implication algebras

Theorem 4.2. $[14$ Let $\mathcal{E}$ be a regular category. The following statements are equivalent:

(i) $\mathcal{E}$ is a Goursat category;

(ii) $\forall R, S \in \operatorname{Equiv}(X), R \vee S=R S R(=S R S) \in \operatorname{Equiv}(X)$; 
(iii) every relation $P \rightarrow X \times Y$ in $\mathcal{E}, P P^{\circ} P P^{\circ}=P P^{\circ}$;

(iv) every reflexive relation $F$ in $\mathcal{E}, F^{\circ} F=F F^{\circ} \in \operatorname{Equiv}(X)$;

$(v)$ every reflexive and positive relation $R=U^{\circ} U$ in $\mathcal{E}$ is an equivalence relation;

(vi) $E^{\circ} \leqslant E E$, for any reflexive relation $E$;

(vii) $\left(1_{X} \wedge T\right) T^{\circ}\left(1_{X} \wedge T\right) \leqslant T T$, for any relation $T$ on an object $X$.

Proposition 4.3. Let $\mathcal{E}$ be a regular Goursat categories.

(1) The little desarguesian theorem (2.2) holds in $\mathcal{E}$ when $S_{1}$ is a reflexive relation and $S_{2}, R$ and $T$ are equivalence relations.

(2) The Closure Lemma 1 (2.1) holds in $\mathcal{E}$ when $S_{1}$ is a reflexive relation and $S_{2}, R$ and $T$ are equivalence relations.

(3) The Closure Lemma 2 (2.3) holds in $\mathcal{E}$ when $S_{1}$ is a reflexive relation and $S_{2}, R$ and $T$ are equivalence relations.

(4) The Little Pappian Theorem holds true in $\mathcal{E}$ when $S_{1}$ is a reflexive relation and $S_{2}, R$ and $T$ are equivalence relations.

(5) The Closure Lemma 3 holds true in $\mathcal{E}$ when $S$ is a reflexive relation and $R$ and $T$ are equivalence relations.

Proof. The proof is based on Proposition 3.4 in [15] which stipule that a Goursat category satisfies the Shifting Lemma when $S_{1}$ is a reflexive relation and $R$ and $T$ are equivalence relations.

In a regular context, it suffices to give a proof in set-theoretical terms (see Metatheorem 2.12 and [27], for instance). We show that the Little Desarguesian Theorem holds true in $\mathcal{E}$.

Let $R, S_{1}, S_{2}$ and $T$ be equivalence relations on the same object $X$ in $\mathcal{E}$ such that $R \wedge S_{i} \leqslant T$ and assume that $x, y, z, x^{\prime}, y^{\prime}, z^{\prime}$, are related as in 2.2. The pairs 
$(y, y),\left(y^{\prime}, y^{\prime}\right),(x, z),\left(x^{\prime}, z^{\prime}\right)$ are elements of $S_{1}$. Defining equivalence relations $P, Q$ and $F$ on $S_{1}$ by:

$$
\begin{aligned}
(u, v) P(r, s) & \Leftrightarrow v S_{2} s \\
(u, v) Q(r, s) & \Leftrightarrow u T r \text { and } v S_{2} s \\
(u, v) F(r, s) & \Leftrightarrow u R r \text { and } v R s
\end{aligned}
$$

We obtain

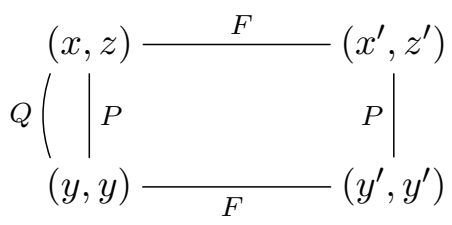

If we want to apply the Shifting Lemma we have to check that $F \wedge P \leq Q$. Thus, let $(a, b) F \wedge P(c, d)$. First of all $(a, b) \in S_{1}$ and $(c, d) \in S_{1}$. Moreover $(b, d) \in R \wedge S_{2}$. Hence $(b, d) \in T$ by assumption (Proposition 3.4 in [15]). Hence

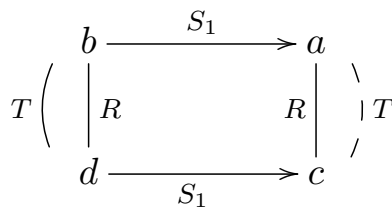

We may apply the Shifting Lemma to

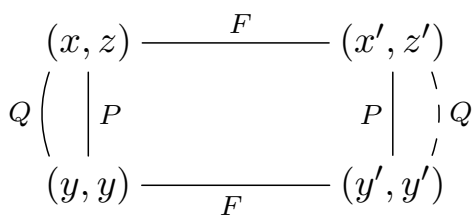

We conclude that $\left(x^{\prime}, z^{\prime}\right) Q\left(y^{\prime}, y^{\prime}\right)$ and, consequently, that $\left(x^{\prime}, y^{\prime}\right) \in T$.

Theorem 4.4. Let $\mathcal{E}$ be a regular category and consider the following assertions. 
(1) $\mathcal{E}$ is a Goursat category;

(2) the little desarguesian theorem (2.2) holds in $\mathcal{E}$ when $S_{1}, S_{2}$ are reflexive relations and $R$ and $T$ are reflexive and positive relations;

(3) The Escher Cube, (2.1) holds in $\mathcal{E}$ when $S_{1}, S_{2}$ are reflexive relations and $R$ and $T$ are reflexive and positive relations;

(4) The Closure Lemma 1, 2.3) holds in $\mathcal{E}$ when $S_{1}, S_{2}$ are reflexive relations and $R$ and $T$ are reflexive and positive relations;

(5) the Little Pappian Theorem holds true in $\mathcal{E}$ when $S_{1}, S_{2}$ are reflexive relations and $R$ and $T$ are reflexive and positive relations;

(6) the Closure Lemma 3 holds true in $\mathcal{E}$ when $S_{1}, S_{2}$ are reflexive relations and $R$ and $T$ are reflexive and positive relations.

Then

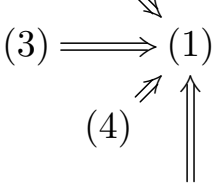

Proof. $(2) \Rightarrow(1)$ We shall prove that for any reflexive relation $E$ on $X$ in $\mathcal{E}, E E^{\circ}=$ $E^{\circ} E$ (see Theorem 4.2 (iv)). Suppose that $(x, y) \in E E^{\circ}$. Then, for some $z$ in $X$, one has that $(z, x) \in E$ and $(z, y) \in E$. Consider the reflexive and positive relations $R=E E^{\circ}$ and $T=E^{\circ} E$, and the reflexive relation $E$ on $X$. Then we have:

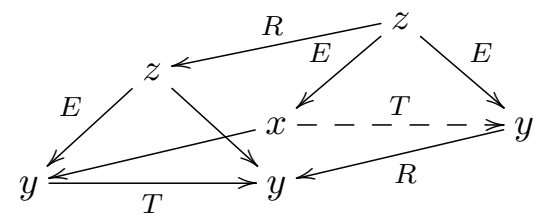


to conclude that $(x, y) \in E^{\circ} E$. The equality $E^{\circ} E=E E^{\circ}$ follows immediately. (3) $\Rightarrow$ (1) Obvious, (4) $\Rightarrow(1)$ With the assumption of previous proof, we obtain

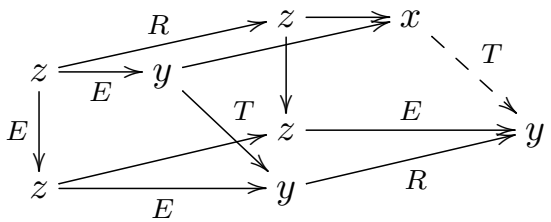

Similarly $(5) \Rightarrow(1)$ is obvious, $(6) \Rightarrow(1)$ we observe with $S_{1}=S_{2}=E$

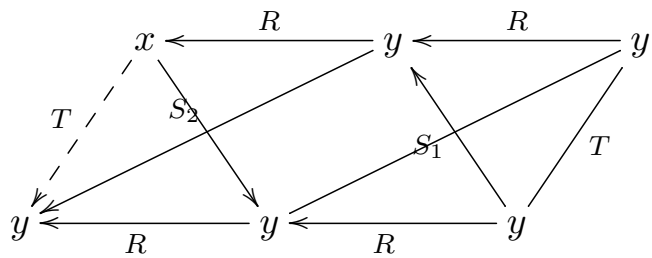

A variety $\mathcal{V}$ of universal algebras is called 3-permutable when the strictly weaker equality $R S R=S R S$ holds. Such varieties are characterized by the existence of two quaternary operations $p$ and $q$ satisfying the identities $p(x, y, y, z)=x, p(u, u, v, v)=$ $q(u, u, v, v), q(x, y, y, z)=z$. We start by giving a direct proof of Little Desarguesian theorem in the varietal context. We give an alternative proof which is suitable to be extended to the categorical context of regular categories.

Theorem 4.5. Let $\mathcal{E}$ be a majority and 3-permutable varieties.Let $x, y, z, x^{\prime}, y^{\prime}, z^{\prime}$, be elements of $X$ and $R, T, S_{1}, S_{2}$ congruences on $X$ with $R \wedge S_{i} \leqslant T$, for $i=1,2$. Then the diagram (2.2) holds in $\mathcal{E}$.

Proof. Let $R, S_{1}, S_{2}$ and $T$ be congruence on the same algebra $X$ in $\mathcal{E}$ such that $R \wedge S_{i} \leqslant$ $T$ and assume that $x, y, z, x^{\prime}, y^{\prime}, z^{\prime}$, are related as in 2.2. From the relations

$$
\begin{array}{ll}
x T x R x^{\prime} S_{1} S_{2} x^{\prime} & x T x R x S_{1} S_{2} x \\
x T x R x^{\prime} S_{1} S_{2} y^{\prime} & x T x R x S_{1} S_{2} y \\
x T y R y^{\prime} S_{1} S_{2} y^{\prime} & x T x R y S_{1} S_{2} y \\
y T y R y^{\prime} S_{1} S_{2} y^{\prime} & y T y R y S_{1} S_{2} y
\end{array}
$$


we may deduce the following ones by applying the quaternary operations $p$ and $q$, respectively:

$$
p(x, x, x, y) T p(x, x, y, y) R p\left(x^{\prime}, x^{\prime}, y^{\prime}, y^{\prime}\right) S p\left(x^{\prime}, y^{\prime}, y^{\prime}, y^{\prime}\right)
$$

and

$$
q(x, x, x, y) T q(x, x, y, y) R q\left(x^{\prime}, x^{\prime}, y^{\prime}, y^{\prime}\right) S q\left(x^{\prime}, y^{\prime}, y^{\prime}, y^{\prime}\right)
$$

one has $x T p(x, x, y, y) R p\left(x^{\prime}, x^{\prime}, y^{\prime}, y^{\prime}\right) S x^{\prime}$ and $y T q(x, x, y, y) R q\left(x^{\prime}, x^{\prime}, y, y\right) S y^{\prime}$; one has $x \operatorname{TxR} R(x, x, y, y) S x$ and $y \operatorname{TyR} q(x, x, y, y) S y$.

Since $\mathcal{E}$ is majority variety, then $R \wedge S_{1} S_{2} \leqslant\left(R \wedge S_{1}\right)\left(R \wedge S_{2}\right) \leqslant T T=T$. We apply the Shifting Lemma to
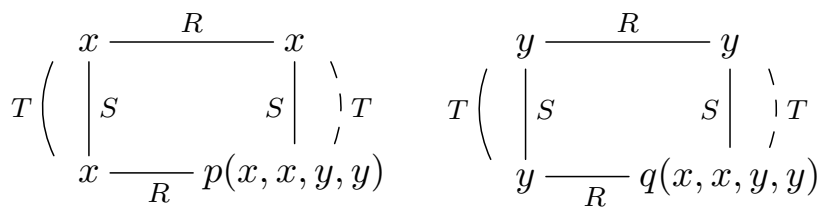

Again, we apply the Shifting Lemma to

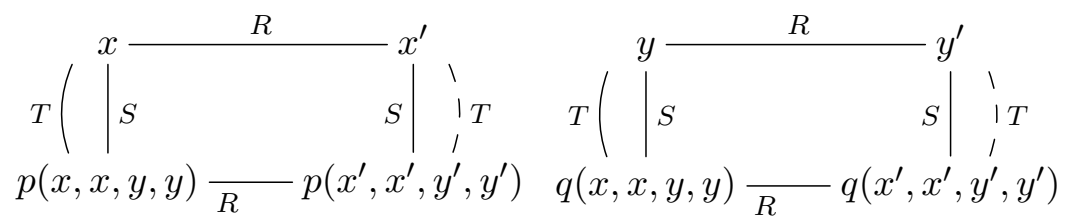

we obtain $x^{\prime} T p\left(x^{\prime}, x^{\prime}, y^{\prime}, y^{\prime}\right)=q\left(x^{\prime}, x^{\prime}, y^{\prime}, y^{\prime}\right) T y^{\prime}$; it follows that $\left(x^{\prime}, y^{\prime}\right) \in T$.

We adapt this varietal proof into a categorical one using an appropriate matrix and the corresponding relations which may be deduced from it (see [16] for more details). The kind of matrix we use translates the quaternary identities into the property on relations given in Theorem 4.2 (iii):

$$
\left(\begin{array}{llll|ll}
x & y & y & z & x & z \\
u & u & v & v & \alpha & \alpha
\end{array}\right)
$$

Thus, the introduction of a new element $\alpha$, to represent the identity $p(u, u, v, v)=$ $q(u, u, v, v)(=\alpha)$. 
For this matrix, the interpretation gives: for any binary relation $P$, if $x P u, y P u, y P v$ and $z P v$, then $x P \alpha$ and $z P \alpha$, for some $\alpha$; this gives the property $P P^{o} P P^{o} \leq P P^{o}$ Since $P P^{o} \leq P P^{o} P P^{o}$ is always true, we get precisely $P P^{o} P P^{o}=P P^{o}$ from Theorem 4.2(iii).

Theorem 4.6. Let $\mathcal{E}$ be a regular majority and Goursat categories. Let $x, y, z, x^{\prime}, y^{\prime}, z^{\prime}$, be elements of $X$ and $R, T, S_{1}, S_{2}$ are equivalence relations on $X$ with $R \wedge S_{i} \leqslant T$, for $i=1,2$. Then the diagram (2.2) holds in $\mathcal{E}$.

Proof. We extend the proof of Theorem 4.5 to a categorical context by constructing an appropriate matrix as follows

$$
\left(\begin{array}{cccc|cc}
x & x & x & y & x & y \\
x & y & y & y & x & y \\
x^{\prime} & y^{\prime} & y^{\prime} & y^{\prime} & x^{\prime} & y^{\prime} \\
& & & & & \\
x & x & y & y & \theta & \theta \\
x^{\prime} & x^{\prime} & y^{\prime} & y^{\prime} & \omega & \omega
\end{array}\right)
$$

We define a relation $P$ from $X^{3}$ to $X^{2}$ by:

$$
(a, b, c) P(d, e) \Leftrightarrow \exists l \text { such that } a T d R l S_{1} S_{2} b, \quad l R e, e S_{1} S_{2} c \text { and } a S_{1} S_{2} d .
$$

From the matrix we see that $\left(x, x, x^{\prime}\right) P\left(x, x^{\prime}\right),\left(x, y, y^{\prime}\right) P\left(x, x^{\prime}\right),\left(x, y, y^{\prime}\right) P\left(y, y^{\prime}\right)$ and $\left(y, y, y^{\prime}\right) P\left(y, y^{\prime}\right)$, we also see that $\left(x, x, x^{\prime}\right) P P^{\circ} P P^{\circ}\left(y, y, y^{\prime}\right)$ from which we conclude that $\left(x, x, x^{\prime}\right) P P^{\circ}\left(y, y, y^{\prime}\right)$. It then follows that $\left(x, x, x^{\prime}\right) P(\theta, \omega)$ and $\left(y, y, y^{\prime}\right) P(\theta, \omega)$, for some $(\theta, \omega)$. Let $S=S_{1} S_{2}$, i.e., there exist $\alpha$ and $\beta$ such that

$$
\begin{aligned}
& x T \theta R \alpha S x, \alpha R \omega, \quad \omega S x^{\prime} \text { and } x S \theta \\
& y T \theta R \beta S y, \quad \beta R \omega, \quad \omega S y^{\prime} \text { and } y S \theta
\end{aligned}
$$

Since $\mathcal{E}$ is majority category, $R \wedge S_{1} S_{2} \leqslant\left(R \wedge S_{1}\right)\left(R \wedge S_{2}\right) \leqslant T T=T$. We apply the Shifting Lemma to

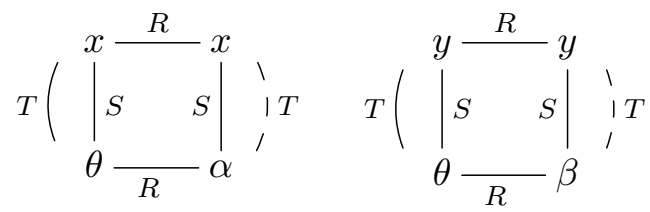


Again, we apply the Shifting Lemma to
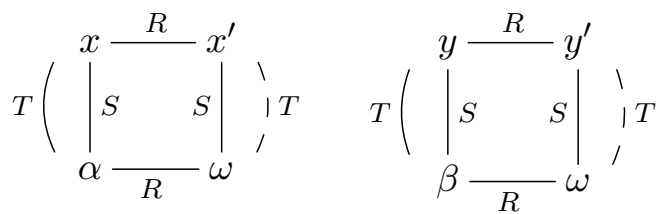

we obtain $x^{\prime} T \omega T y^{\prime}$; it follows that $\left(x^{\prime}, y^{\prime}\right) \in T$.

\section{Equivalence Relations in $n$-permutable Categories}

In this section we investigate the category $\operatorname{Equiv}(\mathcal{E})$ of internal equivalence relations in a regular category $\mathcal{E}$. We show that $\operatorname{Equiv}(\mathcal{E})$ is a $2 n+1$-permutable category whenever $\mathcal{E}$ is.

For $n=1$ (see [17]) $\mathcal{E}$ be a Goursat category. Then the category $\operatorname{Equiv}(\mathcal{E})$ is a also a Goursat category.

Following [1], given any pair $(R, S)$ of reflexive relations on an object $X$ in a regular category $\mathcal{E}$, let us denote by $(R ; S)_{n} ; n \geq 2$ the alternate composition $R S R S \cdots$ of length $n$ which is a reflexive relation as well. Clearly we have: $(R, S)_{n} \leq(R, S)_{n+1}$ and $(S, R)_{n} \leq(R, S)_{n+1}$. Then call $n$-permutable a regular category satisfying $(R, S)_{n}=$ $(S, R)_{n}$ for all pairs $(R, S)$ of equivalence relations.

Theorem 5.1. ([24], Theorem 3.5 of [9]) Let $n \geq 2$ and let $\mathcal{E}$ be a regular category. The following statements are equivalent:

(i) $\mathcal{E}$ is n-permutable category;

(ii) $\left(P, P^{\circ}\right)_{n+1} \leq\left(P, P^{\circ}\right)_{n-1}$ for any relation $P$;

(iii) $(R, S)_{n}$ is an equivalence relation and is therefore $R \vee S$;

(iv) $\left(1_{X} \wedge T\right) T^{\circ}\left(1_{X} \wedge T\right) \leq T^{n-1}$, for any relation $T$ on an objet $X$;

(v) for any reflexive endorelation $E \longmapsto X \times X$ in $\mathcal{E}$, the relation $\left(E, E^{\circ}\right)_{n-1}$ is an equivalence relation; 
(vi) for any such reflexive endorelation $E$, the relation $\left(E, E^{\circ}\right)_{n-1}$ is transitive;

(vii) for any such reflexive endorelation $E$ we have $\left(E, E^{\circ}\right)_{n-1}=\left(E^{\circ}, E\right)_{n-1}$;

(viii) $E^{\circ} \leq E^{n-1}$ for any reflexive relation $E$.

Proposition 5.2. [4] Given any $2 n$-permutable or $(2 n+1)$-permutable regular category $\mathcal{E}$, any regular epimorphism $f: X \rightarrow Y$ and any equivalence relation $S$ on $X$, then the reflexive relation $f(S)^{n}$ is an equivalence relation in $\mathcal{E}$.

Another characterization of $(2 n+1)$-permutable categories in terms of (equivalence) relations is given by the preservation of equivalence relations through the regular image by a regular epimorphism as follows:

Theorem 5.3. [4] Given any regular category $\mathcal{E}, T F A E$ :

(i) the regular category $\mathcal{E}$ is $(2 n+1)$-permutable.

(ii) for any regular epimorphism $f: X \rightarrow Y$ in $\mathcal{E}$ and any equivalence relation $S$ on $X$, then $f(S)^{n}$ is an equivalence relation.

The kernel pair of a morphism $(f, g)$ in $\operatorname{Equiv}(\mathcal{E})$ is given by the kernel pairs $E q(f)$ of $f$ and $E q(g)$ of $g$ in $\mathcal{E}$

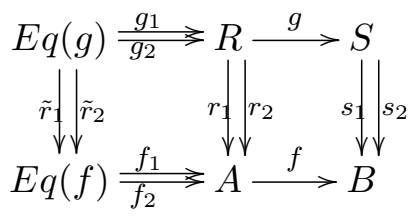

Consequently, a morphism $(f, g)$ is a monomorphism in $\operatorname{Equiv}(\mathcal{E})$ if and only if both $f$ and $g$ are monomorphisms in $\mathcal{E}$. When $\mathcal{E}$ is $(2 n+1)$-permutable category, a similar property holds with respect to regular epimorphisms:

Lemma 5.4. Let $R$ and $S$ be two equivalence relations in a $(2 n+1)$-permutable category $\mathcal{E}$ and $(f, g): R \rightarrow S$ be a morphism

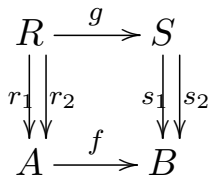


in $\operatorname{Equiv}(\mathcal{E})$. Then $(f, g)$ is a regular epimorphism in $\operatorname{Equiv}(\mathcal{E})$ if and only if both $f$ and $g$ are regular epimorphisms in $\mathcal{E}$.

Proof. When $f$ and $g$ are regular epimorphisms in $\mathcal{E}$, it is not difficult to check that $(f, g)$ is necessarily the coequalizer of its kernel pair in $\operatorname{Equiv}(\mathcal{E})$ given in 5.1 (one uses the fact that $g=\operatorname{coe} q\left(g_{1}, g_{2}\right)$ and $f=\operatorname{coe} q\left(f_{1}, f_{2}\right)$ in $\left.\mathcal{E}\right)$.

Conversely, let $(f, g)$ be a morphism in $\operatorname{Equiv}(\mathcal{E})$ as in (5.1) that is a regular epimorphism in $\operatorname{Equiv}(\mathcal{E})$. Consider the kernel pairs of $f$ and $g$, the (regular epimorphism, monomorphism) factorization $f=i q$ of $f$, and the regular image $\left(q(R)^{n}, t_{1}, t_{2}\right)$ of $\left(R, r_{1}, r_{2}\right)$ along $q$. We obtain the following commutative diagram

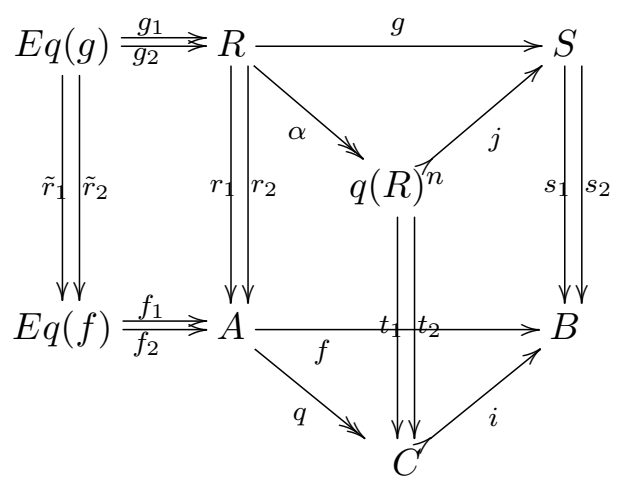

where $\left(q(R)^{n}, t_{1}, t_{2}\right) \in \operatorname{Equiv}(\mathcal{E})$ (by Theorem 5.3) and $(i, j)$ is the morphism in $\operatorname{Equiv}(\mathcal{E})$ such that $(i, j)(q, \alpha)=(f, g)$. Note that $j$ is induced by the fact that $(i \times i)\left\langle t_{1}, t_{2}\right\rangle \alpha$ is the (regular epimorphism, monomorphism) factorization of $\left\langle s_{1}, s_{2}\right\rangle g$, thus it is a monomorphism.

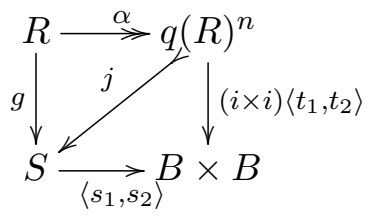

From the fact that $(f, g)$ is the coequalizer of its kernel pair in $\operatorname{Equiv}(\mathcal{E})$ and that $(q, \alpha)$ is the coequalizer of $\left(f_{1}, g_{1}\right)$ and $\left(f_{2}, g_{2}\right)$ in $\operatorname{Equiv}(\mathcal{E})$ (since $(i, j)$ is a monomorphism), 
it easily follows that $(i, j)$ is an isomorphism in $\operatorname{Equiv}(\mathcal{E})$. This implies that $f$ and $g$ are regular epimorphisms in $\mathcal{E}$.

Corollary 5.5. Let $\mathcal{E}$ be a $(2 n+1)$-permutable category. Then the category Equiv $(\mathcal{E})$ is a regular category.

Theorem 5.6. Equiv $(\mathcal{E})$ is a $(2 n+1)$-permutable category whenever $\mathcal{E}$ is.

Proof. Let $\left(R,\left(p_{1}, q_{1}\right),\left(p_{2}, q_{2}\right)\right)$ be an equivalence relation on $\left(S, s_{1}, s_{2}\right)$ in the category $\operatorname{Equiv}(\mathcal{E})$ and $f=\left(f_{1}, f_{2}\right)$ a regular epimorphism in $\operatorname{Equiv}(\mathcal{E})$. We must prove that $f(R)^{n}$ is an equivalence relation in $\operatorname{Equiv}(\mathcal{E})$. The relation $f(R)^{n}$ is obtained through the following diagram

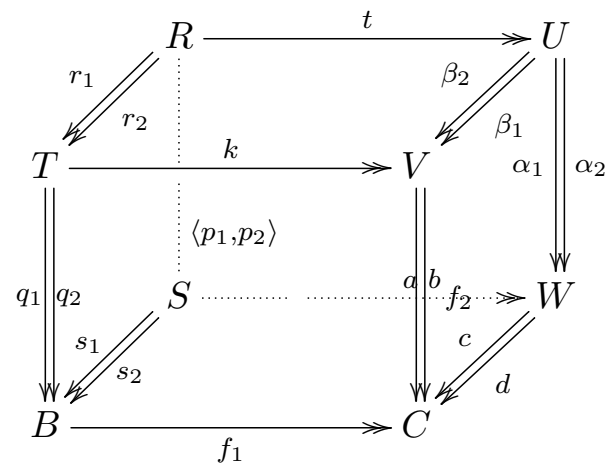

where $U=f_{2}(R)^{n}$ and $V=f_{1}(T)^{n}$ are in $\operatorname{Equiv}(\mathcal{E})$.

One has: $a k r_{1}=c \alpha_{1} t$ and $b k r_{1}=c \alpha_{2} t$, thus the following diagram commutes:

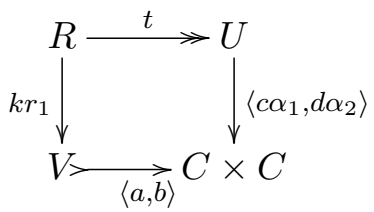

Since $t$ is a strong epimorphism and $\langle a, b\rangle$ is a monomorphism, there exists $\beta_{1}: U \rightarrow V$ such that $\langle a, b\rangle \beta_{1}=\left\langle c \alpha_{1}, d \alpha_{2}\right\rangle$. The existence of $\beta_{2}$ is obtained similarly. Since

$$
(\langle a, b\rangle \times\langle a, b\rangle)\left\langle\beta_{1}, \beta_{2}\right\rangle=\langle c \times c, d \times d\rangle\left\langle\alpha_{1}, \alpha_{2}\right\rangle,
$$


it follows that $\left\langle\beta_{1}, \beta_{2}\right\rangle: U \longmapsto V \times V$ is a monomorphism and thus $\left(U, \beta_{1}, \beta_{2}\right)$ is a relation on $V$. All parallel morphisms of the left face represent equivalence relations and all horizontal morphisms are regular epimorphisms, so that all parallel morphisms of the right face also represent equivalence relations (Theorem 5.3), and then $f(R)^{n}$ is an equivalence relation in $\operatorname{Equiv}(\mathcal{E})$.

\section{References}

[1] B. R. Amougou Mbarga, Triangular scheme revisited in the light of $n$-permutable categories, Earthline Journal of Mathematical Sciences 6(1) (2021), 105-116.

https://doi.org/10.34198/ejms.6121.105116

[2] B. R. Amougou Mbarga, Anticommutativity and $n$-schemes, Earthline Journal of Mathematical Sciences 6(1) (2021), 175-186.

https://doi.org/10.34198/ejms.6121.175186

[3] B.R. Amougou Mbarga, Some remarks on Goursat lemma, Algebraic Structures and their Applications, in Press.

[4] D. Bourn, Internal equivalence relations, modular formula and Goursat condition in non-regular context, Cahiers du L.M.P.A., Published online: http://www-lmpa.univ-littoral.fr/publications/articles/lmpa526.pdf

[5] M. Barr, Exact categories, in: Exact Categories and Categories of Sheaves, 1-120, Lecture Notes in Math., vol. 236, Springer, Berlin, 1971.

https://doi.org/10.1007/BFb0058580

[6] F. Borceux and D. Bourn, Metatheorems, in: Mal'cev, Protomodular, Homological and Semi-Abelian Categories, Mathematics and its Applications, vol. 566, Kluwer Academic Publishers, Dordrecht, 2004.

https://doi.org/10.1007/978-1-4020-1962-3_1

[7] S. Burris and H.P. Sankappanavar, A course in universal algebra, Graduate Texts in Mathematics, 78, Springer-Verlag, New York-Berlin, 1981.

[8] A. Carboni, J. Lambek and M. C. Pedicchio, Diagram chasing in Mal'cev categories, Journal of Pure and Applied Algebra 69 (1991), 271-284.

https://doi.org/10.1016/0022-4049(91)90022-T 
[9] A. Carboni, G. M. Kelly and M. C. Pedicchio, Some remarks on Maltsev and Goursat categories, Appl. Categor. Struct. 1 (1993), 385-421. https://doi.org/10.1007/BF00872942

[10] I. Chajda and E.K. Horváth, A scheme for congruence semidistributivity, Discuss. Math. Gen. Algebra Appl. 23 (2003), 13-18. https://doi.org/10.7151/dmgaa.1060

[11] I. Chajda, E.K. Horváth and G. Czédli, Trapezoid lemma and congruence distributivity, Math. Slovaca 53 (2003), 247-253.

[12] J. Lambek, Goursats Theorem and the Zassenhaus Lemma, Canad. J. Math. 10 (1958), 45-56. https://doi.org/10.4153/CJM-1958-005-6

[13] M. Gran, Notes on regular, exact and additive categories, Summer School on Category Theory and Algebraic Topology, Ecole Polytechnique Fédérale de Lausanne, 11-13 September 2014.

[14] M. Gran and D. Rodelo, Beck-Chevalley condition and Goursat categories, 2013. arxiv:1512.04066v1

[15] M. Gran, D. Rodelo and I. T. Nguefeu, Variations of the shifting lemma and Goursat categories, Algebra Univers. 80 (2019), Paper No. 2. https://doi.org/10.1007/s00012-018-0575-z

[16] M. Gran, D. Rodelo and I. Tchoffo Nguefeu, Facets of congruence distributivity in Goursat categories, 2020. https://arxiv.org/abs/1909.10211v2

[17] M. Gran, D. Rodelo and Idriss Tchoffo Nguefeu, Some remarks on connectors and groupoids in Goursat categories, Logical Methods in Computer Science 13(3) (2017), 1-12. https://doi.org/10.23638/LMCS-13(3:14) 2017

[18] B. Jonnson, Algebras whose congruence lattices are distributive, Math. Scand. 21 (1967), 110-121.https://doi.org/10.7146/math.scand.a-10850

[19] J. Hagemann and A. Mitschke, On n-permutable congruences, Algebra Univers. 3 (1973), Article number: 8.https://doi.org/10.1007/BF02945100

[20] M. Hoefnagel, Anticommutativity and the triangular lemma, 2020. arXiv:2008.00486v2

[21] M. Hoefnagel, Majority categories, Theory Appl. Categ. 34 (2019), 249-268. 
[22] M. Hoefnagel, Characterizations of majority categories, Appl. Categor. Struct. 28 (2020), 113-134.https://doi.org/10.1007/s10485-019-09571-z

[23] M. Hoefnagel, A categorical approach to lattice-like structures, Ph.D. thesis, 2018.

[24] P.-A. Jacqmin and D. Rodelo, Stability properties characterising $n$-permutable categories, Theory and Applications of Categories 32(45) (2017), 1563-1587.

[25] K.A. Kearnes and E.W. Kiss, The triangular principle is equivalent to the triangular scheme, Algebra Univers. 54 (2005), 373-383.

https://doi.org/10.1007/s00012-005-1954-9

[26] Mathilde Olivette Ngaha Ngaha, Isomorphism theorems and descent in star-regular categories, Prom.: Gran, Marino. http://hdl.handle.net/2078.1/145867

[27] H. Peter Gumm, Geometrical methods in congruence modular algebras, Mem. Amer. Math. Soc. 45 (1983). http://dx.doi.org/10.1090/memo/0286

[28] H. Peter Gumm, The little Desarguesian theorem for algebras in modular varieties, Proc. Amer. Math. Soc. 80 (1980), 393-397.

https://doi.org/10.1090/s0002-9939-1980-0580991-6

This is an open access article distributed under the terms of the Creative Commons Attribution License (http://creativecommons.org/licenses/by/4.0/), which permits unrestricted, use, distribution and reproduction in any medium, or format for any purpose, even commercially provided the work is properly cited. 